Few-Body Systems 0, 1-6 (2014)

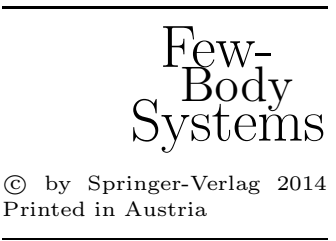

\title{
Light nuclei from chiral EFT interactions
}

\author{
P. Navrátil ${ }^{1 *}$, V. G. Gueorguiev ${ }^{1}$, J. P. Vary ${ }^{1,2}$, W. E. Ormand ${ }^{1}$, A. Nogga ${ }^{3}$, \\ S. Quaglioni ${ }^{1}$ \\ ${ }^{1}$ Lawrence Livermore National Laboratory, L-414, P.O. Box 808, Livermore, CA 94551, USA \\ ${ }^{2}$ Department of Physics and Astronomy, Iowa State University, Ames, Iowa 50011, USA \\ ${ }^{3}$ Forschungszentrum Jülich, Institut für Kernphysik (Theorie), D-52425 Jülich, Germany
}

\begin{abstract}
Recent developments in nuclear theory allow us to make a connection between quantum chromodynamics (QCD) and low-energy nuclear physics. First, chiral effective field theory $(\chi \mathrm{EFT})$ provides a natural hierarchy to define two-nucleon (NN), three-nucleon (NNN), and even four-nucleon interactions. Second, ab initio methods have been developed capable to test these interactions for light nuclei. In this contribution, we discuss ab initio no-core shell model (NCSM) calculations for $s$-shell and $p$-shell nuclei with $\mathrm{NN}$ and NNN interactions derived within $\chi \mathrm{EFT}$.
\end{abstract}

\section{Introduction}

The major outstanding problem in nuclear physics is to calculate properties of finite nuclei starting from the basic interactions among nucleons. There are two aspects to this problem. First, the basic interactions among nucleons are complicated, they are not uniquely defined and there is evidence that more than just two-nucleon forces are important. Second, the nuclear many-body problem is very difficult to solve. This is a direct consequence of the complex nature of the inter-nucleon interactions.

Interactions among nucleons are governed by QCD. In the low-energy regime relevant to nuclear structure, QCD is non-perturbative, and, therefore, hard to solve. Thus, theory has been forced to resort to models for the interaction. New theoretical developments, however, allow us connect QCD with low-energy nuclear physics through promising bridge of $\chi \mathrm{EFT}[1$.

\section{Ab initio no-core shell model}

In the $a b$ initio NCSM, we consider a system of $A$ point-like non-relativistic nucleons that interact by realistic $\mathrm{NN}$ or $\mathrm{NN}+\mathrm{NNN}$ interactions. Unlike in standard

${ }^{*}$ E-mail address:navratil1@llnl.gov 
shell model calculations, in the NCSM there is no inert core, all the nucleons are considered active. Therefore the "no-core" in the name of the approach. Besides the employment of realistic $\mathrm{NN}$ or $\mathrm{NN}+\mathrm{NNN}$ interactions, two other major features characterize the NCSM: i) the use of an harmonic oscillator (HO) basis truncated by a chosen maximal HO excitation energy $N_{\max } \hbar \Omega$ relative to the unperturbed ground state of the $A$-nucleon system; ii) the use of effective interactions. The reason behind the choice of the $\mathrm{HO}$ basis is the fact that this is the only basis that allows to use single-nucleon coordinates and consequently the second-quantization representation without violating the translational invariance of the system. The powerful techniques based on the second quantization and developed for standard shell model calculations can then be utilized. Therefore the "shell model" in the name of the approach. As a downside, one has to face the consequences of the incorrect asymptotic behavior of the HO basis. The second feature comes as a consequence of the basis truncation. In order to speed up convergence with the basis enlargement, we construct an effective interaction from the original realistic $\mathrm{NN}$ or $\mathrm{NN}+\mathrm{NNN}$ potentials by means of a unitary transformation. The effective interaction depends on the basis truncation and by construction recovers the original realistic $\mathrm{NN}$ or $\mathrm{NN}+\mathrm{NNN}$ interaction as the size of the basis approaches infinity. In principle, one can also perform calculations with the unmodified, "bare", original interactions. Such calculations are then variational with respect to the HO frequency $\Omega$ and the basis truncation parameter $N_{\max }$.

\section{Light nuclei from chiral EFT interactions}

Currently the most promising approach to the construction of accurate internucleon forces from QCD is the $\chi$ EFT. The $\chi$ EFT predicts, along with the NN interaction at the leading order, an NNN interaction starting at the 3rd order (next-to-next-to-leading order or $\mathrm{N}^{2} \mathrm{LO}$ ) [1, 2], and even an NNNN interaction starting at the 4 th order $\left(\mathrm{N}^{3} \mathrm{LO}\right)[3$. The details of QCD dynamics are contained in parameters, low-energy constants (LECs), not fixed by the symmetry. These parameters can be constrained by experiment. A crucial feature of $\chi \mathrm{EFT}$ is the consistency between the NN, NNN and NNNN parts. As a consequence, at $\mathrm{N}^{2} \mathrm{LO}$ and $\mathrm{N}^{3} \mathrm{LO}$, except for two LECs, assigned to two NNN diagrams, the potential is fully constrained by the parameters defining the NN interaction.

We adopt the potentials of the $\chi \mathrm{EFT}$ at the orders presently available, the $\mathrm{NN}$ at $\mathrm{N}^{3} \mathrm{LO}$ of Ref. [4] and the NNN interaction at $\mathrm{N}^{2} \mathrm{LO}$ [2]. We use ab initio NCSM calculations in two ways. One of them is the determination of the LECs assigned to two NNN diagrams, $c_{D}$ and $c_{E}$ [5] that must be determined in $A \geq 3$ systems. $c_{D}\left(c_{E}\right)$ is the strength of the $N N-\pi-N(N N N)$ contact term. The other is testing predictions of the chiral NN and NNN interactions for light nuclei.

It is important to note that our NCSM results through $A=4$ are fully converged in that they are independent of the $N_{\max }$ cutoff and the $\hbar \Omega \mathrm{HO}$

energy. This is demonstrated in Fig. 1, where convergence of the ${ }^{4} \mathrm{He}$ ground-state energy using $\chi \mathrm{EFT}$ interactions with and without the NNN terms is shown. For heavier systems, we characterize the approach to convergence by the dependence 


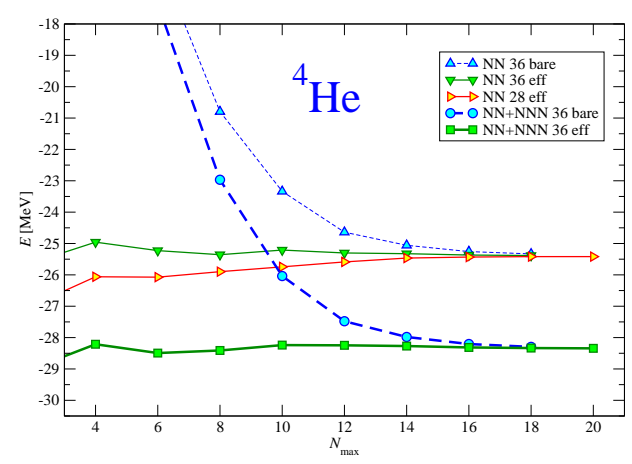

Figure 1. ${ }^{4} \mathrm{He}$ ground-state energy dependence on the size of the basis. The HO frequencies of $\hbar \Omega=28$ and $36 \mathrm{MeV}$ were employed. Results with (thick lines) and without (thin lines) the NNN interaction are shown. The full lines correspond to calculations with threebody effective interaction, the dashed lines to calculations with the bare interaction.

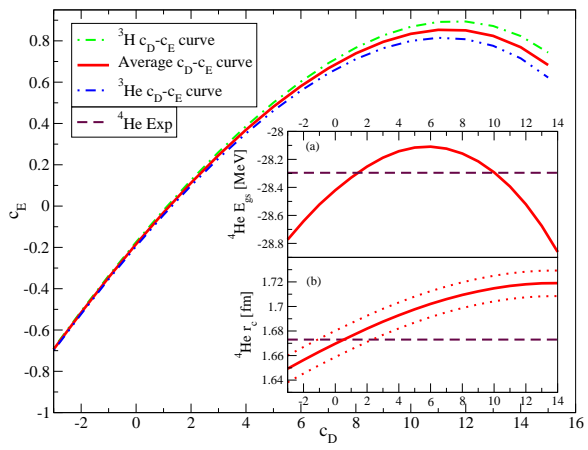

Figure 2. Relations between $c_{D}$ and $c_{E}$ for which the binding energy of ${ }^{3} \mathrm{H}(8.482 \mathrm{MeV})$ and ${ }^{3} \mathrm{He}(7.718 \mathrm{MeV})$ are reproduced. (a) ${ }^{4} \mathrm{He}$ ground-state energy along the averaged curve. (b) ${ }^{4} \mathrm{He}$ charge radius $r_{c}$ along the averaged curve. Dotted lines represent the $r_{c}$ uncertainty due to the uncertainties in the proton charge radius.

of results on $N_{\max }$ and $\hbar \Omega$.

Fig. 2 shows the trajectories of the two LECs $c_{D}-c_{E}$ that are determined from fitting the binding energies of the $A=3$ systems. Separate curves are shown for ${ }^{3} \mathrm{H}$ and ${ }^{3} \mathrm{He}$ fits, as well as their average. There are two points where the binding of ${ }^{4} \mathrm{He}$ is reproduced exactly. We observe, however, that in the whole investigated range of $c_{D}-c_{E}$, the calculated ${ }^{4} \mathrm{He}$ binding energy is within a few hundred $\mathrm{keV}$ of experiment. Consequently, the determination of the LECs in this way is likely not very stringent. We therefore investigate the sensitivity of the $p$-shell nuclear properties to the choice of the $c_{D}-c_{E}$ LECs. First, we maintain the $A=3$ binding energy constraint. Second, we limit ourselves to the $c_{D}$ values in the vicinity of the point $c_{D} \sim 1$ since the values close to the point $c_{D} \sim 10$ overestimate the ${ }^{4} \mathrm{He}$ radius.

While most of $p$-shell nuclear properties, e.g. excitation spectra, are not very sensitive to variations of $c_{D}$ in the vicinity of the $c_{D} \sim 1$ point, we were able to identify several observables that do demonstrate strong dependence on $c_{D}$. For example, the ${ }^{6} \mathrm{Li}$ quadrupole moment that changes sign depending on the choice of $c_{D}$. In Fig. 3, we display the ratio of the B(E2) transitions from the ${ }^{10} \mathrm{~B}$ ground state to the first and the second $1^{+} 0$ state. This ratio changes by several orders of magnitude depending on the $c_{D}$ variation. This is due to the fact that the structure of the two $1^{+} 0$ states is exchanged depending on $c_{D}$. From Figs. 2 and 3, we can see that for $c_{D}<-2$ the ${ }^{4} \mathrm{He}$ radius underestimate experiment while for $c_{D}>0$ the lowest two $1^{+}$states of ${ }^{10} \mathrm{~B}$ are reversed. We therefore select $c_{D}=-1$ for our further investigation.

We present in Fig. 4 the excitation spectra of ${ }^{10} \mathrm{~B}$ as a function of $N_{\max }$ for the chiral NN interaction alone. The convergence with increasing $N_{\max }$ is quite reasonable for the low-lying states. Similar convergence rates are obtained 
4

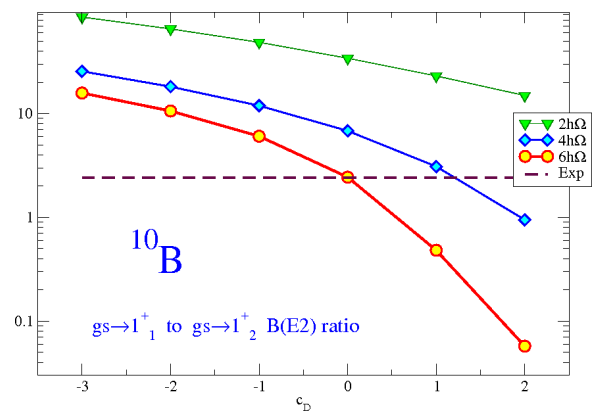

Figure 3. Dependence of the ${ }^{10} \mathrm{~B}$ $\mathrm{B}\left(\mathrm{E} 2 ; 3_{1}^{+} 0 \rightarrow 1_{1}^{+} 0\right) / \mathrm{B}\left(\mathrm{E} 2 ; 3_{1}^{+} 0 \rightarrow 1_{2}^{+} 0\right)$ ratio on $c_{D}$, with $c_{E}$ constrained by the $A=3$ binding energy fit, for different basis sizes. The HO frequency of $\hbar \Omega=14 \mathrm{MeV}$ was employed.
Light nuclei from chiral EFT interactions

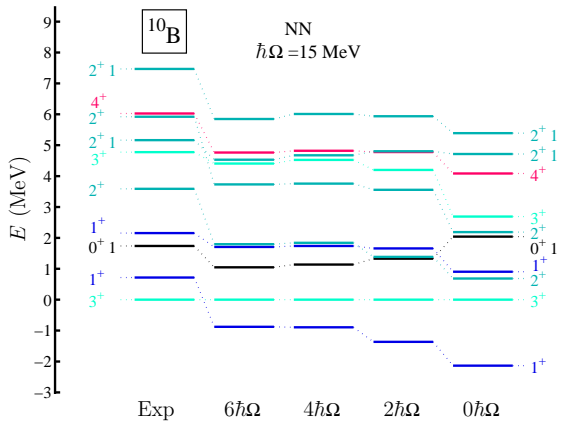

Figure 4. ${ }^{10} \mathrm{~B}$ excitation spectra as function of the basis space size $N_{\max }$ at $\hbar \Omega=15 \mathrm{MeV}$ using the chiral $\mathrm{NN}$ interaction compared to experiment. The isospin of the states not explicitly depicted is $T=0$.

for other $p$-shell nuclei that we investigate. A remarkable feature of the ${ }^{10} \mathrm{~B}$ results is the observation that the chiral $\mathrm{NN}$ interaction alone predicts incorrect ground-state spin of ${ }^{10} \mathrm{~B}$. The experimental value is $3^{+} 0$, while the calculated one is $1^{+} 0$. On the other hand, once we also include the chiral NNN interaction in the Hamiltonian, which is actually required by the $\chi \mathrm{EFT}$, the correct ground-state spin is predicted. Further, once we select the $c_{D}$ value as discussed above, i.e. $c_{D}=-1$, we also obtain the two lowest $1^{+} 0$ states in the experimental order.

We display in Fig. 5 the natural parity excitation spectra of four nuclei in the middle of the $p$-shell with both the $\mathrm{NN}$ and the $\mathrm{NN}+\mathrm{NNN}$ interactions from $\chi \mathrm{EFT}$. The results shown are obtained in the largest basis spaces achieved to date for these nuclei with the NNN interactions, $N_{\max }=6(6 \hbar \Omega)$. Overall, the NNN interaction contributes significantly to improve theory in comparison with experiment. This is especially well-demonstrated in the odd mass nuclei for the lowest few excited states. The case of the ground state spin of ${ }^{10} \mathrm{~B}$ and its sensitivity to the presence of the NNN interaction discussed also in Fig. 4, is clearly evident. We note that the ${ }^{10} \mathrm{~B}$ results shown in Fig. 4 were obtained with the $\mathrm{HO}$ frequency of $\hbar \Omega=15 \mathrm{MeV}$, while those in Fig. 5 with $\hbar \Omega=14 \mathrm{MeV}$. A weak HO frequency dependence of the $N_{\max }=6$ results is apparent.

Further details on calculations presented in this section were published in Ref. [6]. These calculations demonstrate that the chiral NNN interaction makes substantial contributions to improving the spectra and other observables. However, there is room for further improvement in comparison with experiment. We used a strength of the $2 \pi$-exchange piece of the NNN interaction, which is consistent with the NN interaction that we employed (i.e. from Ref. [4]). This strength is somewhat uncertain (see e.g. Ref. [5]). Therefore, it will be important to study the sensitivity of our results with respect to this strength. Further on, it will be interesting to incorporate sub-leading NNN interactions and also four-nucleon interactions, which are also order $\mathrm{N}^{3} \mathrm{LO}[3]$. Finally, it will be useful to extend the basis spaces to $N_{\max }=8(8 \hbar \Omega)$ for $A>6$ to further improve convergence. 
P. Navrátil, V. G. Gueorguiev, J. P. Vary, W. E. Ormand, A. Nogga and S. Quaglioni

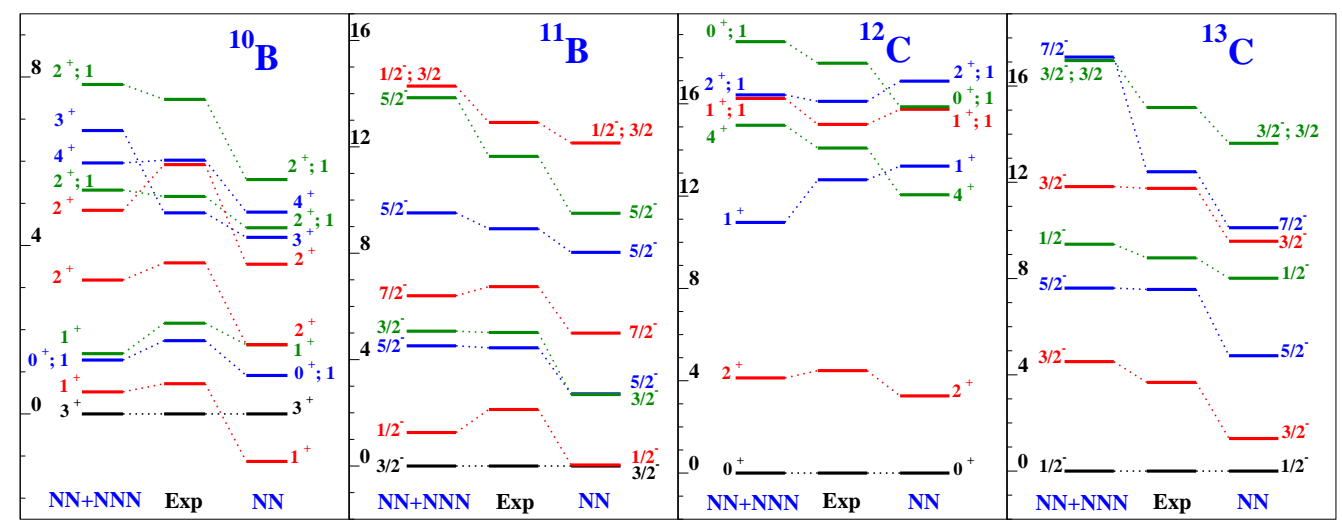

Figure 5. States dominated by $p$-shell configurations for ${ }^{10} \mathrm{~B},{ }^{11} \mathrm{~B},{ }^{12} \mathrm{C}$, and ${ }^{13} \mathrm{C}$ calculated at $N_{\max }=6$ using $\hbar \Omega=15 \mathrm{MeV}\left(14 \mathrm{MeV}\right.$ for $\left.{ }^{10} \mathrm{~B}\right)$. Most of the eigenstates are isospin $T=0$ or $1 / 2$, the isospin label is explicitly shown only for states with $T=1$ or $3 / 2$. The excitation energy scales are in $\mathrm{MeV}$.

\section{Beyond nuclear structure with chiral EFT interactions}

A shortcoming of the $a b$ initio NCSM is its incorrect description of long-range correlations and its lack of coupling to continuum due to the expansion of the eigenstates in a finite HO basis. If we want to build upon the ab initio NCSM to microscopically describe nuclear reactions we can proceed in two ways. First, we can rely on techniques such as the Lorentz Integral Transform that reduce the continuum problem to a bound-state-like problem. Using this approach, we performed ${ }^{4} \mathrm{He}$ photo-disintegration cross section calculations using the $\chi \mathrm{EFT}$ interactions [7]. We demonstrated a sizeable effect of the chiral NNN interaction on the cross section. Second, we can augment the ab initio NCSM basis by explicitly including cluster states and solve for their relative motion while imposing the proper boundary conditions. This approach, applicable to a wide range of reactions as well as to weakly bound states, is in the spirit of the resonating group method (RGM) [8]. In our approach, we use the ab initio NCSM wave functions for the clusters involved and effective interactions derived from realistic NN (and eventually also from NNN) potentials. As an example, a converged calculation of the $\mathrm{n}+{ }^{4} \mathrm{He}{ }^{2} S_{1 / 2}$ phase shift using the $\chi \mathrm{EFT} \mathrm{NN}$ interaction is presented in Fig. 6. More details on this approach are given in Ref. [9].

\section{Conclusions}

The $a b$ initio NCSM evolved into a powerful many-body technique. Presently, it is the only method capable to use interactions derived within the $\chi \mathrm{EFT}$ for systems of more than four nucleons. Among its successes is the demonstration of the importance of the NNN interaction for nuclear structure. Applications to nuclear reactions with a proper treatment of long-range properties are under development. Extension to heavier nuclei is achieved through the importancetruncated NCSM [10]. Within this approach, ab initio calculations for nuclei as 


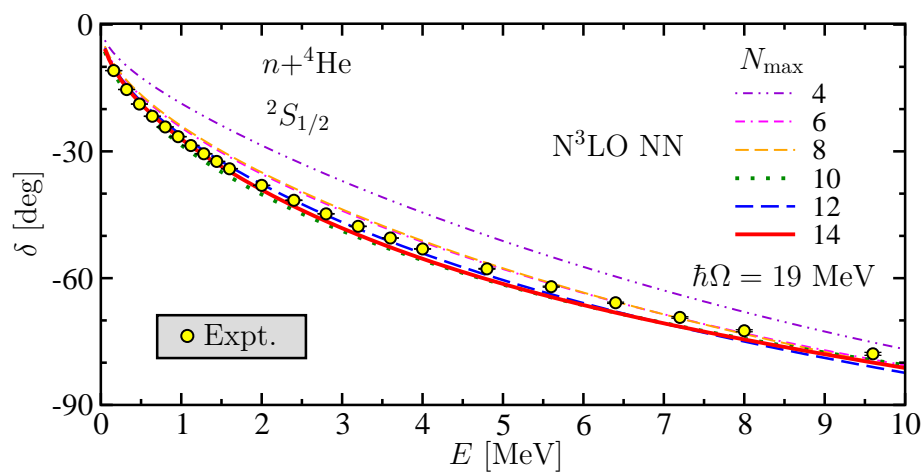

Figure 6. The calculated ${ }^{2} S_{1 / 2}$ phase shift for the $\mathrm{n}+{ }^{4}$ He system compared with experimental data. The $\chi \mathrm{EFT}$ NN potential of Ref. 4] was used in model spaces up to the $14 \hbar \Omega$.

heavy as ${ }^{40} \mathrm{Ca}$ become possible.

Acknowledgement. This work performed under the auspices of the U.S. Department of Energy by Lawrence Livermore National Laboratory under Contract DE-AC52-07NA27344. Support from U.S. DOE/SC/NP (Work Proposal Number SCW0498) and the Department of Energy under Grant DE-FC02-07ER41457 is acknowledged.

\section{References}

1. S. Weinberg, Physica 96A, 327 (1979); Phys. Lett. B 251, 288 (1990); Nucl. Phys. B363, 3 (1991); J. Gasser et al., Ann. of Phys. 158, 142 (1984); Nucl. Phys. B250, 465 (1985).

2. U. van Kolck, Phys. Rev. C 49, 2932 (1994); E. Epelbaum, A. Nogga, W. Glöckle, H. Kamada,Ulf-G. Meissner and H. Witala, Phys. Rev. C 66, 064001 (2002).

3. E. Epelbaum, Phys. Lett. B 639, 456 (2006).

4. D. R. Entem and R. Machleidt, Phys. Rev. C 68, 041001(R) (2003).

5. A. Nogga, P. Navrátil, B. R. Barrett and J. P. Vary Phys. Rev. C 73, 064002 (2006).

6. P. Navrátil and V. G. Gueorguiev and J. P. Vary, W. E. Ormand and A. Nogga, Phys. Rev. Lett. 99, 042501 (2007); nucl-th/0701038.

7. S. Quaglioni and P. Navrátil, Phys. Lett. B 652, 370 (2007).

8. Y. C. Tang, M. LeMere and D. R. Thompson, Phys. Rep. 47 (1978) 167; K. Langanke and H. Friedrich, Advances in Nuclear Physics, chapter 4., Plenum, New York, 1987.

9. S. Quaglioni and P. Navrátil, arXiv:0712.0855.

10. R. Roth and P. Navrátil, Phys. Rev. Lett 99, 092501 (2007). 